\title{
Topical Delivery of Four Neuroprotective Ingredients by Ethosome-Gel: Synergistic Combination for Treatment of Oxaliplatin-Induced Peripheral Neuropathy
}

This article was published in the following Dove Press journal:

International Journal of Nanomedicine

\author{
Hong-mei Lin ${ }^{1} * *$ \\ Long-fei Lin ${ }^{2, *}$ \\ Ming-yi Sun ${ }^{3}$ \\ Jia $\mathrm{Liu}^{3}$ \\ Qing $W_{u^{3}}$
}

'Beijing Research Institute of Chinese Medicine, Beijing University of Chinese Medicine, Beijing 100029, People's Republic of China; ${ }^{2}$ Institute of Chinese Materia Medica, China Academy of Chinese Medical Sciences, Beijing 100700, People's Republic of China; ${ }^{3}$ Department of TCM Pharmaceutics, School of Chinese Materia Medica, Beijing University of Chinese Medicine, Beijing 100029, People's Republic of China

*These authors contributed equally to this work
Correspondence: Qing Wu

Department of TCM Pharmaceutics,

School of Chinese Materia Medica, Beijing

University of Chinese Medicine, Beijing

100029, People's Republic of China

Tel +861084738603

Fax +86 I0 847386II

Email qwu@vip.sina.com
Background: Peripheral neuropathy is a common and painful side effect that occurs in patients with cancer induced by Oxaliplatin (OXL). The neurotoxicity correlates with the damage of dorsal root ganglion (DRG) neurons and Schwann cells (SCs). Hydroxysafflor yellow A (HSYA), icariin, epimedin B and 3, 4-dihydroxybenzoic acid (DA) are the main neuroprotective ingredients identified in Wen-Luo-Tong (WLT), a traditional Chinese medicinal topical compound. The purpose of this study was to prepare and evaluate the efficacy of an ethosomes gel formulation loaded with a combination of HSYA, icariin, epimedin B and DA. However, the low $\log P$ value, poor solubility and macromolecule are several challenges for topical delivery of these drugs.

Methods: Ethosomes were prepared by the single-step injection technique. Particle size, entrapment efficiency and in vitro drug deposition studies were determined to select the optimum ethosomes. The optimized ethosomes were further incorporated into carbopol to obtain a gel. The rheological properties, morphology, in vitro drug release, in vitro gel application and skin distribution of the ethosomes gels were studied. A rat model of oxaliplatin-induced neuropathy was established to assess the therapeutic efficacy of the ethosomes gel.

Results: Seventy percent (v/v) ethanol, cinnamaldehyde and Phospholipon 90G were employed to develop ethosomes a carrier system. This system had a high entrapment efficiency, carried large amounts of HSYA, epimedin B, DA and icarrin, and penetrated deep into the epidermis and dermis. The optimized ethosomes had the maximum deposition of icariin, HSYA, epimedin B and relative higher amount of DA in epidermis $\left(2.00 \pm 0.13 \mu \mathrm{g} / \mathrm{cm}^{2}, 5.72\right.$ $\pm 0.75 \mu \mathrm{g} / \mathrm{cm}^{2}, 1.97 \pm 0.27 \mu \mathrm{g} / \mathrm{cm}^{2}$ and $9.25 \pm 1.21 \mu \mathrm{g} / \mathrm{cm}^{2}$, respectively). $0.5 \%$ carbopol 980 was selected to develop the ethosomes gel with desirable viscoelasticity and spreadability, which was suitable for topical application. The mechanical allodynia and hyperalgesia induced by OXL in rats were significantly reduced after the new ethosomes gel was applied to rats compared to model group.

Conclusion: Based on our findings, the ethosomes gel delivery system provided a new formulation for the topical delivery of HSYA, icariin, epimedin B and DA to counteract OXL-induced peripheral neuropathy.

Keywords: hydroxysafflor yellow A, icariin, epimedin B, 3, 4-dihydroxybenzoic acid, DA, oxaliplatin-induced neuropathy, ethosomes gel

\section{Introduction}

Oxaliplatin (OXL) is an effective chemotherapeutic agent that is widely used to treat advanced colorectal cancers. Unfortunately, it often induces severe acute and 
chronic neurotoxicity. The clinical symptoms of OXLinduced chronic neuropathy include pain, dysesthesia, sensory loss and sensorimotor dysfunction, which significantly impair the quality of life and frequently result in a premature suspension of treatment and decreased survival. ${ }^{1}$ Many medications and physical modalities have been used to prevent and treat OXL-induced peripheral neuropathy (OIPN). Nevertheless, the treatment of OIPN symptoms remains a challenge and additional research is needed to intervene OIPN symptoms., ${ }^{2,3}$ Although the detailed mechanism of OIPN remains unclear, recent reports indicated a correlation between the neurotoxicity and oxidative stress and mitochondrial dysfunction in dorsal root ganglion (DRG) neurons, inducing apoptosis in DRG neurons and the degeneration of intraepidermal nerve fibers (IENF), particularly in the limbs. ${ }^{2,4,5}$ Schwann cells (SCs) are an essential component of peripheral nerve fibers that nurture to axons and exert a neuroprotective effect by promoting axon regeneration and myelination. ${ }^{6,7}$ Based on the findings described above, the application of neuroprotective agents to SCs and DRG neurons injured by OXL may represent an effective treatment that prevents and counteracts OIPN.

A therapy combining two or more active agents has been regarded as one of the most powerful strategies to increase the therapeutic effect by acting on multi-targets. ${ }^{8}$ Active agents present in and identified from natural chemicals are gradually gaining credibility and becoming effective approaches. ${ }^{9}$ An effective method to discover the active agents in the Chinese compound formulas used to treat diseases is currently unavailable. As shown in our previous studies, hydroxysafflor yellow A (HSYA), icariin, epimedin $B$ and 3,4-dihydroxybenzoic acid (DA) significantly increase the viability of the primary cultured SCs injured by OXL. They were the main neuroprotective ingredients identified in the topical formulation of Wen-luo-tong used to treat peripheral neuropathy induced by OXL using ultra-performance liquid chromatography coupled with a quadrupole time-offlight mass spectrometry (UPLC-Q-TOF/MS). The formulation consists of Herba Epimedii (Epimedium sagittatum), Herba Erodii (Geranium wilfordiiMaxim), Safflower (Carthamustinctorius L.) and Herba Cassia Twig (Cinnamomum cassia Presl). ${ }^{10,11}$ Icariin and epimedin $\mathrm{B}$ are the main active ingredients of Herba Epimedii and promote remyelination and inhibit endoplasmic reticulum stress-induced neuronal apoptosis. ${ }^{12-15}$ HSYA presents in safflower and exerts neurotrophic and anti-inflammatory effect against ischemic stroke. ${ }^{16,17} \mathrm{DA}$ is an active ingredient of Herba Erodii with a neuroprotective effect. ${ }^{18}$ The combination of icariin, epimedin B, HSYA and DA exerted an excellent protective effect on SCs and DRG neurons, which was better than the individual components in our previous study.

The OXL-induced degeneration of IENF, which is derived from DRG neurons, is the main reason related to the key symptoms, such as pain and numbness. ${ }^{19}$ In addition, the axons of DRG neurons retrogradely transport mitochondria, lysosomes and nanoparticles to cell bodies from neuron terminals. ${ }^{20-22}$ In this context, a topical formulation can be used to deliver the four active agents to the IENF and DRG cell bodies, providing a new therapeutic method to treat OIPN. The nanodelivery systems have gained remarkable recognition due to their promising potential advantages including targeted delivery, stability, and solubility as well as potentiate therapeutic. These advanced delivery systems include liposomes, ethosomes, nanoparticles, nanoemulsion, and polymeric nanoparticles (NPs). ${ }^{23}$ Thus, we will develop a novel topical nanodelivery system co-loaded with HSYA, icariin, epimedin B and DA to counteract OIPN that has the ability to deliver these agents to deep skin layers (dermal/epidermal junction). The new formulation would not only repair the impaired IENFs in the epidermis by protecting SCs but also deliver these agents to the cell bodies of DRG neurons and prevent OXL-induced DRG apoptosis through transport along the axon. However, several challenges have been noted in preparing a topical formulation to deliver HSYA, epimedin $\mathrm{B}$ and icariin into the skin. HSYA has been proven to be water-soluble with a $\log P$ value of $-4.33 .{ }^{24}$ The molecular weight of epimedin B is 808.7. ${ }^{10}$ Icariin displays a poor solubility both in water and lipid solutions, ${ }^{25}$ which limited the selection of the appropriate delivery systems. The saturation solubility of icarrin in different solvent was tested in our previous studies such as oleic acid, isopropyl myristate, Labrafac 1349, 0.2\% TWEEN 80, 0.2\% Brij 58 and different percentage ethanol. Icarrin had a superior solubility in $70 \%(\mathrm{v} / \mathrm{v})$ ethanol $(2.899 \pm 0.083 \mathrm{mg} / \mathrm{mL})$. Therefore, ethosomes were selected as the delivery systems in this work, which was prepared by $70 \%(\mathrm{v} / \mathrm{v})$ ethanol.

Ethosomes are lipid-based nanocarriers that contain cholesterol, ethanol or propylene glycol, and have been widely used for transdermal delivery. The high concentration of ethanol in ethosomes increases vesicular deformability and allows ethosomes able to intactly squeeze through the stratum corneum to the deep layers of skin. Furthermore, macromolecules and various hydrophilic and 
hydrophobic drugs have also been effectively co-loaded in ethosomes. This formulation is extremely suitable for incorporating multi-components of traditional Chinese medicine. Nevertheless, the topical application of ethosomes is suffers from a low contact time with the skin due to the liquid status. ${ }^{26-30}$

The main objective of the present study is primarily to optimize the ethosomes by varying independent variables, such as the cinnamaldehyde, phospholipon $90 \mathrm{G}$, ethanol and propylene glycol concentrations, for the topical delivery of HSYA, icariin, epimedin B and DA. Furthermore, the optimized formulation was incorporated into gel matrices to provide ease of application and increase the retention time on skin as a drug reservoir. The rheological and skin targeting properties of the ethosomes gel were characterized, and its effectiveness in the management of OIPN was evaluated.

\section{Materials and Methods Materials}

HSYA (purity $>90 \%$ ) was provided by Chengdu University (Chengdu, China). Icariin (batch number 489-32-7, purity $>98 \%$ ) was purchased from Shyuanye Biotechnology Co., Ltd (Shanghai, China). Cinnamaldehyde (batch number FY1265VX53J, purity $>98 \%$ ) and 3, 4-dihydroxybenzoic acid (batch number FY17830102, purity $>98 \%$ ) were obtained from Nantong Feiyu Biotechnology Co., Ltd (Nantong, China). Phospholipon 90G, Lipoid 75 andNatipide II were gifts from Lipoid GmbH (Newark, NJ, USA). Soybean lecithin was purchased from TCI America (Boston, MA, USA). Oleic acid (OA), isopropyl myristate (IPM), Tween 20, and Span 80 were purchased from Sigma (Saint Louis, MO, USA). Labrosol ALF, Labrafac 1349, Transcutol P were gifts from GATTEFOSSE (Paramus, NJ, USA). Phosphate buffer saline (PBS), Brij58, ethanol, propylene glycol, acetonitrile, and phosphoric acid were purchased from ACROS Organics (Newark, NJ, USA). All other ingredients and reagents were of analytical grade. Cadaver skin from the posterior torso (female, aged 56) was supplied by the NewYork Firefighters Skin Bank.

\section{HPLC Method}

The analytical method designed to simultaneously quantify the levels of HSYA, icariin, epimedin B, DA and cinnamaldehyde were performed by using an Agilent 1100 HPLC (Agilent 1100, Palo Alto, CA). Samples were filtered and analyzed on a reverse-phase $\mathrm{C}_{8}$ column (Agilent $\mathrm{XDB}-\mathrm{C} 8,150 \times 4.6 \mathrm{~mm}, 5 \mu \mathrm{m}$, Palo Alto, CA) at $30^{\circ} \mathrm{C}$.
The gradient elution program was employed with methanol as solvent $\mathrm{A}$ and a $0.2 \%$ phosphoric acid solution as solvent $\mathrm{B}$. The following gradient program was as follows: 0-3min, 20\% A; 3-6min, 20-55\% A; 6-15min, 55\% A; and $15-18 \mathrm{~min}, 55-20 \% \mathrm{~A}$. The flow rate was set at to $1 \mathrm{~mL} / \mathrm{min}$. Samples were analyzed at 270 and $403 \mathrm{~nm}$. Stock solutions of the five compounds were prepared in $70 \%$ methanol. Calibration solutions of HSYA, icariin, epimedin B and DA were prepared at concentrations ranging from 0.25 to $200 \mu \mathrm{g} / \mathrm{mL}$. Cinnamaldehyde (CA) was prepared at concentrations ranging from $0.5-200 \mu \mathrm{g} / \mathrm{mL}$. The retention time of DA, CA, epimedin B and icariin were $4.025,10.526,11.511$ and $12.676 \mathrm{~min}$ under $270 \mathrm{~nm}$, respectively. The retention time of HSYA was $7.187 \mathrm{~min}$ under 403nm.

\section{Preparation of Ethosomes}

Ethosomes were prepared using the single-step injection technique. $^{31}$ Icariin, epimedin $\mathrm{B}$, cholesterol and Phospholipon 90G were dissolved in different volumes of $70 \%(\mathrm{v} / \mathrm{v})$ ethanol and propylene glycol. The mixtures were maintained at $32 \pm 2^{\circ} \mathrm{C}$ with constant magnetic stirring (Benchmark, Sayreville, NJ) at $700 \mathrm{rpm}$ for 2 h. Afterwards, an appropriate amount of CA was added and the mixture was continually stirred for $0.5 \mathrm{~h}$. HSYA and DA were dissolved in double-distilled water and slowly added into the mixture by using a microinjection pump (Chemyx Fusion 200, Stafford, TX) at a rate of $0.2 \mathrm{~mL} / \mathrm{min}$. The resulting mixture was stirred at $700 \mathrm{rpm}$ for $5 \mathrm{~min}$ to obtain the ethosomes. Ethosomes were subjected to sonication at $4{ }^{\circ} \mathrm{C}$ using a probe sonicator (Branson SFX150, Branson, MI) at a 50\% amplitude for $1 \mathrm{~min}$ (pulse $10 \mathrm{~s}$ and stop $5 \mathrm{~s}$ ) to decrease the particle size. The ethosomes were optimized for different formulation variables including the amounts of CA, Phospholipon $90 \mathrm{G}$ and cholesterol, and ratio of $70 \%(\mathrm{v} / \mathrm{v})$ ethanol and propylene glycol. The compositions for optimizing the amount of CA are reported in Table 1. After optimizing the amount of $\mathrm{CA}$, the compositions for optimizing the amount of Phospholipon 90G, cholesterol, the ratio of $70 \%$ (v/v) ethanol and propylene glycol are reported in Table 2.

\section{Characterization and Optimization of Ethosomes \\ Particle Size}

The mean particle size and polydispersity index of the ethosomes were analyzed using the dynamic light scattering 
Table I Optimization of the Amount of Cinnamaldehyde

\begin{tabular}{|c|c|c|c|c|c|c|c|c|c|c|}
\hline \multirow[t]{2}{*}{ Batches } & \multirow{2}{*}{$\begin{array}{l}\text { Phospholipon90G } \\
\text { (mg) }\end{array}$} & \multirow{2}{*}{$\begin{array}{l}70 \% \\
\text { Ethanol } \\
(\mathrm{mL})\end{array}$} & \multirow{2}{*}{$\begin{array}{l}\text { PG } \\
(\mathrm{mL})\end{array}$} & \multirow{2}{*}{$\begin{array}{l}\text { Cholesterol } \\
(\mathrm{mg})\end{array}$} & \multirow{2}{*}{$\begin{array}{l}\text { Icarrin } \\
(\mathrm{mg})\end{array}$} & \multirow{2}{*}{$\begin{array}{l}\text { CA } \\
(\mu L)\end{array}$} & \multirow{2}{*}{$\begin{array}{l}\text { Epimedin } \\
\text { B (mg) }\end{array}$} & \multirow{2}{*}{$\begin{array}{l}\text { HSYA } \\
(\mathrm{mg})\end{array}$} & \multirow{2}{*}{$\begin{array}{l}\text { DA } \\
(\mathrm{mg})\end{array}$} & \multirow{2}{*}{$\frac{\text { Water }}{(\mathrm{mL})}$} \\
\hline & & & & & & & & & & \\
\hline I & 150 & 2.1 & 0.3 & - & 4.0 & 0 & 4.0 & 5.0 & 5.0 & 3.6 \\
\hline 2 & 150 & 2.1 & 0.3 & - & 4.0 & 2 & 4.0 & 5.0 & 5.0 & 3.6 \\
\hline 3 & 150 & 2.1 & 0.3 & - & 4.0 & 5 & 4.0 & 5.0 & 5.0 & 3.6 \\
\hline 4 & 150 & 2.1 & 0.3 & - & 4.0 & 10 & 4.0 & 5.0 & 5.0 & 3.3 \\
\hline
\end{tabular}

Table 2 Optimization of Formulation Parameters for Ethosomes Preparation

\begin{tabular}{|c|c|c|c|c|c|c|c|c|c|c|}
\hline Batches & $\begin{array}{l}\text { Phospholipon90G } \\
\text { (mg) }\end{array}$ & $\begin{array}{l}70 \% \\
\text { Ethanol } \\
(\mathrm{mL})\end{array}$ & $\begin{array}{l}\text { PG } \\
(\mathrm{mL})\end{array}$ & $\begin{array}{l}\text { Cholesterol } \\
\text { (mg) }\end{array}$ & $\begin{array}{l}\text { Icarrin } \\
(\mathrm{mg})\end{array}$ & $\begin{array}{l}\text { CA } \\
(\mu L)\end{array}$ & $\begin{array}{l}\text { Epimedin } \\
\text { B (mg) }\end{array}$ & $\begin{array}{l}\text { HSYA } \\
(\mathrm{mg})\end{array}$ & $\begin{array}{l}\text { DA } \\
(\mathrm{mg})\end{array}$ & $\begin{array}{l}\text { Water } \\
(\mathrm{mL})\end{array}$ \\
\hline I & 150 & 2.1 & 0.3 & - & 4.0 & 2 & 4.0 & 5.0 & 5.0 & 3.6 \\
\hline 2 & 150 & 2.4 & 0.6 & - & 4.0 & 2 & 4.0 & 5.0 & 5.0 & 3.0 \\
\hline 3 & 150 & 2.4 & 0.6 & 1.0 & 4.0 & 2 & 4.0 & 5.0 & 5.0 & 3.0 \\
\hline 4 & 150 & 2.4 & 0.3 & 1.0 & 4.0 & 2 & 4.0 & 5.0 & 5.0 & 3.3 \\
\hline 5 & 180 & 2.1 & 0.3 & - & 4.0 & 2 & 4.0 & 5.0 & 5.0 & 3.6 \\
\hline 6 & 180 & 2.4 & 0.6 & - & 4.0 & 2 & 4.0 & 5.0 & 5.0 & 3.0 \\
\hline 7 & 180 & 2.4 & 0.6 & 1.0 & 4.0 & 2 & 4.0 & 5.0 & 5.0 & 3.0 \\
\hline 8 & 180 & 2.4 & 0.3 & 1.0 & 4.0 & 2 & 4.0 & 5.0 & 5.0 & 3.3 \\
\hline 9 & 210 & 2.1 & 0.3 & - & 4.0 & 2 & 4.0 & 5.0 & 5.0 & 3.6 \\
\hline 10 & 210 & 2.4 & 0.6 & - & 4.0 & 2 & 4.0 & 5.0 & 5.0 & 3.0 \\
\hline II & 210 & 2.4 & 0.6 & 1.0 & 4.0 & 2 & 4.0 & 5.0 & 5.0 & 3.0 \\
\hline 12 & 210 & 2.4 & 0.3 & 1.0 & 4.0 & 2 & 4.0 & 5.0 & 5.0 & 3.3 \\
\hline
\end{tabular}

technique with a Beckman Coulter instrument (Delsa ${ }^{\mathrm{TM}}$ NanoS). The sample was diluted 10 -fold with $25 \%(\mathrm{v} / \mathrm{v})$ ethanol. Size measurements were conducted at a scattering angle of $90^{\circ}$ at $25 \pm 2^{\circ} \mathrm{C}$. All observations were recorded in triplicate for each formulation.

\section{Entrapment Efficiency}

The entrapment efficiencies (EE) of the five compounds were determined using an ultra-filtration method. ${ }^{32}$ First, $0.5 \mathrm{~mL}$ of ethosomes were placed in an Amicon ${ }^{\circledR}$ ultra-4 centrifugal filter unit (3000 MWCO, Millipore centrifugal filters, Millipore, State) and centrifuged at $5000 \mathrm{rpm}$ for 10 min. The free drug present in the filtrate was diluted to $1 \mathrm{~mL}$ with methanol and analyzed using HPLC (as described in Section 2.2). The EE was calculated using Eq. (1):

$$
\mathrm{EE} \%=\frac{\mathrm{De}-\mathrm{Df}}{\mathrm{De}} \times 100 \%
$$

where $\mathrm{D}_{\mathrm{e}}$ represents the total amount of drug in the ethosomes and $D f$ represents amount of free drug in the filtrate.

\section{In vitro Drug Deposition Studies}

Particle size was considered an important parameter to select the optimum ethosomes dispersion, but it was not the only important parameter. Therefore, all formulations were analyzed using in vitro skin deposition studies to select the optimum formulation, that would deliver the highest amounts of the agents into the skin. Dermatomed human cadaver skin was defrosted slowly and cut into small pieces. The skin samples were placed on Franz diffusion cells (LOGAN FDC-24, Somerset, NJ) with effective diffusion areas of $0.64 \mathrm{~cm}^{2}$. The stratum corneum was placed facing the donor compartment. Each receptor compartment was filled with $5 \mathrm{~mL}$ of freshly prepared $2 \%$ (v/w) Brij 58 to provide sink conditions. Ethosomes formulation $(0.5 \mathrm{~mL})$ were added into the donor compartment of the diffusion cell with a temperature maintained at $37 \pm$ $0.5^{\circ} \mathrm{C}$, and the solution in the receptor compartment was stirred with a magnetic bar at $300 \mathrm{rpm}$. Each set of experiments was performed in triplicate. After $24 \mathrm{~h}$, samples $(0.3 \mathrm{~mL})$ were withdrawn from the receptor compartment and then analyzed using HPLC after filtration through 
a $0.45 \mu \mathrm{m}$ filter membrane. The study was performed under nonocclusive conditions.

Excess ethosomes formulations were wiped from the surface of the skin, and the skin was rinsed by excess ethanol to eliminate the drug remained on the surface of the skin. The epidermis and dermis of all skin samples were separated using the microscopic tweezer. The skin samples were measured after the water on the surface was removed and cut into small pieces. A solution of $70 \%(\mathrm{v} / \mathrm{v})$ methanol $(1 \mathrm{~mL})$ and the pieces of skin were added to a prefilled BeadBug tube and homogenized using BeadBug homogenizer (BeadBug ${ }^{\mathrm{TM}}$ Microtube homogenizer, D1030) at the maximum speed for 3 cycles and 3 min for one cycle. The tube was placed in an ultrasonicator (Crest, Ewing, NJ) for 1 $\mathrm{h}$ and then the solution was filtered through a $0.45 \mu \mathrm{m}$ filter membrane to ensure that all drugs had been extracted from the skin pieces. The concentration of the five agents were determined using HPLC (as described in Section 2.2). The amount of drug in the receptor compartment was calculated using Eq. (2):

$$
Q_{R}=\left(C_{24} \times V\right) / A
$$

The amount of drug deposited in the skin was calculated using Eq.(3).

$$
Q_{S}=(\mathrm{C} \times V) / \mathrm{A}
$$

where $Q_{R}$ represents the cumulative amount of drug that permeated through the skin $\left(\mu \mathrm{g} / \mathrm{cm}^{2}\right), Q_{S}$ represents the amount of drug in the skin $\left(\mu \mathrm{g} / \mathrm{cm}^{2}\right)$, A represents the effective diffusion area and $\mathrm{V}$ represents the volume of methanol in the tube.

\section{Preparation of Ethosomes Gels}

Based on the characterization, the optimum formulation, Batch 6 was used to develop ethosomes gels with carbopol as the gelling agent. Carbopol $(2 \% \mathrm{w} / \mathrm{w})$ was initially dispersed in distilled water and swelled overnight to form a transparent hydrogel. The carbopol hydrogel was neutralized by adding triethanolamine, and then the optimized ethosomes suspension was added with continuous stirring at $300 \mathrm{rpm}$ to obtain a homogeneous ethosomes gel. The rheological properties of carbopol grades (C940 and C980) at two polymer concentration levels $(0.4 \%$ and $0.5 \% \mathrm{w} / \mathrm{w})$ were studied to optimize ethosomes gel.

\section{Optimization of Ethosomes Gels} Rheological Studies

The rheological properties of ethosomes gels were studied to determine if they displayed non-Newtonian properties. The ethosomes contained ethanol, which might affect the crosslinking of the carbopol hydrogel. Viscosity was determined at a range of various shear rates $0.01-500(1 / \mathrm{s})$ using a parallel-plate rheometer (Anton paar, Germany) at a skin temperature of $32{ }^{\circ} \mathrm{C}$. Flow behavior of ethosomes gels was determined by constructing a flow curve and analyzed using the Ostwald model.

$$
\tau=\mathrm{K} \gamma^{\mathrm{n}}
$$

where $\mathrm{n}$ represents the index of flow, $\mathrm{K}$ represents the index of consistency, $\tau$ represents the shear stress, and $\gamma$ represents the shear rate.

\section{Spreadability}

Proper viscosity and spreadability are important properties for a topical gel because they can prolong skin retention time and promote easy smearing during application. The spreadability of the gel formulations was evaluated using the modified parallel plate method at room temperature. ${ }^{32}$ Four hundred-milligram sample was placed on the center of the plate, and the sample was covered by a clean glass panel with a known weight $(10 \mathrm{~g})$. Additional weights were added to the glass panel at intervals of $20 \mathrm{~s}$. Diameters of the spreading areas of the gel formulations were measured in two perpendicular directions. The spreadability factor $\left(\mathrm{S}_{\mathrm{f}}\right)$ of the gel was calculated using Eq.(4).

$$
S_{f}=\mathrm{A} / \mathrm{W}
$$

where $\mathrm{S}_{\mathrm{f}}\left(\mathrm{cm}^{2} / \mathrm{g}\right)$ represents the spreadability factor, $\mathrm{A}$ $\left(\mathrm{cm}^{2}\right)$ represents the final spreading area, and $\mathrm{W}(\mathrm{g})$ represents the total weight.

\section{Viscoelastic and Stability Studies}

The viscoelasticity and stability may be influenced by the presence of Phospolipon $90 \mathrm{G}$ and ethanol in the ethosomes. Viscoelastic parameters, including the elastic modulus (storage, $\mathrm{G}^{\prime}$ ), viscous modulus (loss, $\mathrm{G}^{\prime \prime}$ ) and complex viscosity $\left(\eta^{*}\right)$ were evaluated using a rheometer (Anton Paar USA, Ashland, VA, USA) with an angular frequency $(\omega)$ ranging from 500 to 0.05 radians/second at a skin temperature of $32^{\circ} \mathrm{C}$. 


\section{Characterization of Ethosomes Gels Morphology}

The morphology of optimized ethosomes gels was observed using a transmission electron microscope (JEOL JEM1230, Japan) with an accelerating voltage of $80 \mathrm{kv}$. Samples diluted with ethanol solution were dropped on copper grids. The excess sample was removed, and the grid was negatively stained using a small drop of phosphotungstic acid $(2 \% \mathrm{w} / \mathrm{v})$ for $1-2 \mathrm{~min}$. The grid was allowed to dry thoroughly in air and then the sample was analyzed.

\section{In vitro Drug Release Studies}

A dialysis bag (3000 MWCO, Sigma, Ronkonkoma, NY) was used to test the release of the five drugs from the optimized ethosomes and the ethosome gel formulation. ${ }^{33}$ The cellulose acetate membrane was preactivated in double distilled water (molecular weight cutoff $3000 \mathrm{Da}$ ) and placed on the Franz diffusion cell (LOGAN FDC-24, Somerset, NJ) with an effective diffusion area of $0.64 \mathrm{~cm}^{2}$. Ethosomes gel formulations $(200 \mathrm{mg})$ and ethosomes $(0.2 \mathrm{~mL})$ were separately applied to the donor compartment and $5 \mathrm{~mL}$ of freshly prepared $2 \%$ Brij 58 in PBS ( $\mathrm{pH}=7.2 \sim 7.4)$ was added to the receptor compartment. Vials were maintained at $37 \pm 0.2^{\circ} \mathrm{C}$, and stirred with a magnetic bar at $300 \mathrm{rpm}$. The released medium $(0.3 \mathrm{~mL})$ was collected at $0,1,2,4,6,8,18$, and $24 \mathrm{~h}$. At each time point, $0.3 \mathrm{~mL}$ of fresh medium was added to the main medium compartment. The concentrations of HSYA, icariin, epimedin $\mathrm{B}, \mathrm{DA}$ and $\mathrm{CA}$ were analyzed using HPLC (as described in Section 2.2). The concentrations of drug released from the ethosomes gels and ethosomes were calculated using Eq.(5):

$$
Q=\left(\mathrm{C}_{\mathrm{n}} \times V+\sum_{\mathrm{i}=1}^{\mathrm{n}-1} \mathrm{C}_{\mathrm{i}} \times V_{\mathrm{i}}\right) / \mathrm{A}
$$

where $\mathrm{C}_{\mathrm{n}}$ represents the drug concentration in the receptor medium at time $n, C_{i}$ represents the drug concentration in the receptor medium, A represents the effective diffusion area $\left(0.64 \mathrm{~cm}^{2}\right), \mathrm{V}$ indicates the volume of the receptor medium $(5 \mathrm{~mL})$ and $\mathrm{V}_{\mathrm{i}}$ represents the sampling volume at time $\mathrm{i}$.

\section{In vitro Gel Application Studies}

The methods of application the ethosomes gel formulation six were evaluated using in vitro skin deposition studies. The ethosomes gels were applied to the skin using the three methods described below. 1) A small amount of the ethosomes gel was applied to the skin once at $0 \mathrm{~h} .2$ )
A small amount of the ethosomes gel was applied to the skin twice at $0 \mathrm{~h}$ and $12 \mathrm{~h}$. 3) An excess amount of the ethosomes gel was applied to the skin for $24 \mathrm{~h}$. Other procedures were same as described in the in vitro drug deposition studies.

\section{Skin Distribution Studies}

Skin permeability experiments were performed using dyeloaded ethosomes gels, dye-loaded gel solution and blank gel to further analyze the delivery of the ethosomes gel formulation across the skin layers after application using different methods. Icarrin, epimedin B, and CA were replaced with FITC, and HSYA and DA were replaced with rhodamine $\mathrm{B}$ to prepare ethosomes at a concentration of $2.5 \mathrm{mg} / \mathrm{mL}$. Un-entrapped FITC and rhodamine B were separated using a dialysis bag. Afterwards, dye-loaded ethosomes were further incorporated into the carbopol gel. Next, an ethanol solution $(25 \% \mathrm{v} / \mathrm{v})$ containing $2.5 \mathrm{mg} / \mathrm{mL}$ FITC and $2.5 \mathrm{mg} / \mathrm{mL}$ rhodamine B were used to prepare dye-loaded gel solution as a control for comparison.

Dye-loaded ethosomes gels, a dye-loaded gel solution and a blank gel were applied topically to the human cadaver skin, placed on Franz diffusion cells in dark using the three methods described in the in vitro gel application studies. Skin samples were removed after 24 $\mathrm{h}$ and placed in a $4 \%$ paraformaldehyde fixative overnight at $4{ }^{\circ} \mathrm{C}$. The skin samples were then transferred to $20 \% \mathrm{v} / \mathrm{v}$ sucrose for at least $24 \mathrm{~h}$ and frozen in Optimal Cutting Temperature compound solution (OCT) and sliced into 20 $\mu \mathrm{m}$ sections using a microtome (Leica, Buffalo Grove, IL). These skin sections were then examined under a confocal microscope (Zeiss LSM 780, Jena, Germany).

\section{Neuroprotective Efficacy Animals}

The experiments were performed using male Wistar rats (180-200 g, Beijing Vital River Laboratory Animal Technology Co., Ltd, Beijing, China) housed in plastic cages and maintained in a simulated living environment with a temperature of $25 \pm 2^{\circ} \mathrm{C}$, humidity of $50 \pm 10 \%$. Artificial lighting was provided on a fixed $12 \mathrm{~h}$ lightdark cycle, with food and water available ad libitum. All animal procedures were approved by the Ethical Committee of Beijing University of Chinese Medicine, which was in accordance with the Guide for the Care and Use of Laboratory Animals released by the National Institutes of Health. Animals were randomly divided into the following three groups after 1 week of acclimation: 
ethosomes gel group $(\mathrm{n}=10)$, vehicle control group (Control, $\mathrm{n}=10$ ), and model group (OXL, $\mathrm{n}=10$ ).

\section{Oxaliplatin Administration}

OXL (Jiangsu Hengrui Medicine Co., Ltd. Suzhou, China) was dissolved in 5\% glucose. Rats in the ethosomes gel group and OXL group were intraperitoneally (i.p.) injected with OXL ( $4 \mathrm{mg} / \mathrm{kg}$ ) twice weekly for 4 weeks, while rats in the control group were injected with an equal volume of 5\% glucose as a vehicle on the same schedule. In the ethosomes gel group, the ethosomes gels were applied to the paws and tails of rats twice daily. Pain behaviors and the morphology of dorsal root ganglion neurons were examined.

\section{Behavior Test}

Pain assays were performed with the same methods described in previous study. ${ }^{10}$ Briefly, rats were individually placed on an elevated wire mesh grid and allowed to habituate to the apparatus for $15 \mathrm{~min}$ prior to testing. Calibrated von Frey fibers (4 and $15 \mathrm{~g}$, respectively; Yuyan, China) were applied to the plantar surface of the hind paw five times per side. Paw withdrawals were recorded during experiment, and the positive reactions (\%) were calculated. All behavioral assessments were performed by researchers who were blinded to the rat treatment groups.

\section{Nissl Staining of Dorsal Root Ganglion Neurons}

The morphology of dorsal root ganglion (DRG) neurons was analyzed as described previously. ${ }^{10}$ DRGs (L4-L6) of rats were removed after euthanasia and fixed with neutral formalin for $48 \mathrm{~h}$, dehydrated and embedded in paraffin. The DRG tissues were sliced into $3 \mu \mathrm{m}$ thick sections and stained with toluidine blue. The morphology of dorsal root ganglion neurons from rats in each group was observed under a light microscope including neurons with an eccentric nucleus, multiple nucleoli and dyeing conditions of neurons plasma nissl bodies.

\section{Statistical Analysis}

Statistical significance was calculated using an ANOVA, while Student's $t$-test was used to determine any significant differences between test samples and the control. Differences were considered statistically significant when $\mathrm{p}<0.05$. All data were analyzed using analysis of variance (ANOVA) followed by Tukey's test. Values of $\mathrm{p}<0.05$ were considered as significant.

\section{Results and Discussion \\ Preparation and Optimization of Ethosomes}

A schematic illustrating the methods used to ethosomes gel loaded with the four drugs is shown in Figure 1. Icariin exhibited poor solubility preventing it from easily penetrating the stratum corneum and increasing the difficulty of preparing a formulation with a high entrapment efficiency. In our previous study, icarrin displayed a superior solubility in $70 \%(\mathrm{v} / \mathrm{v})$ ethanol $(2.899 \pm 0.083 \mathrm{mg} / \mathrm{mL})$. Therefore, $70 \%(\mathrm{v} / \mathrm{v})$ ethanol was used as the solvent to develop ethosomes and obtain maximum drug loading for icarrin.

$\mathrm{CA}$ is the main active volatile ingredient in Herba Cassia Twig, which can increase local circulation and is not detected using UPLC-Q-TOF when analyzed in skin penetration studies in vitro. ${ }^{10} \mathrm{CA}$ displays enhanced penetration, which would increase the distribution of drugs in the skin. ${ }^{34}$ In our previous studies, 50-25 $\mu \mathrm{M}$ CA induced the apoptosis of primary Schwann cells cultured in vitro. Therefore, $0,2,5$, and $10 \mu \mathrm{L} \mathrm{CA}$ were used to prepare ethosomes in the present study.

All the ethosomes formulations containing CA displayed a significantly increased the amount of Epimedin $\mathrm{B}$ in the receptor compartment, epidermis and dermis, which also increased with the amount of CA (Table 3). However, CA had no effect on the HSYA and icarrin. This finding may be attributed to the increase in the distribution coefficient of the ingredients appropriate $\log \mathrm{P}$ values, such as Epimedin B and DA, in the skin after treatment with formulations containing CA. The amounts of agents delivered by the ethosomes without CA that were detected in the epidermis and dermis were almost consistent with the amounts of agents loaded in ethosomes. We speculated that ethosomes penetrated the stratum corneum by two ways. Firstly, parts of ethosomes were broken on the surface of the stratum corneum and CA was released, increasing the cumulative amounts of the Epimedin B and DA that penetrated into the skin and resulting in the protective effect of drugs on the DRG neurons after transport through the circulation. Second, ethosomes squeezed the intercellular lipids of the stratum corneum and deposited in the deep layers of epidermis and dermis, which contain numerous INEF.

The amount of $\mathrm{CA}$ in the receptor compartment was increased significantly $(64.33 \pm 13.68$ and $20.18 \pm 4.67 \mu \mathrm{g} / \mathrm{mL})$ when the ethosomes were loaded with 5 and $10 \mu \mathrm{L}$ of $\mathrm{CA}$, 


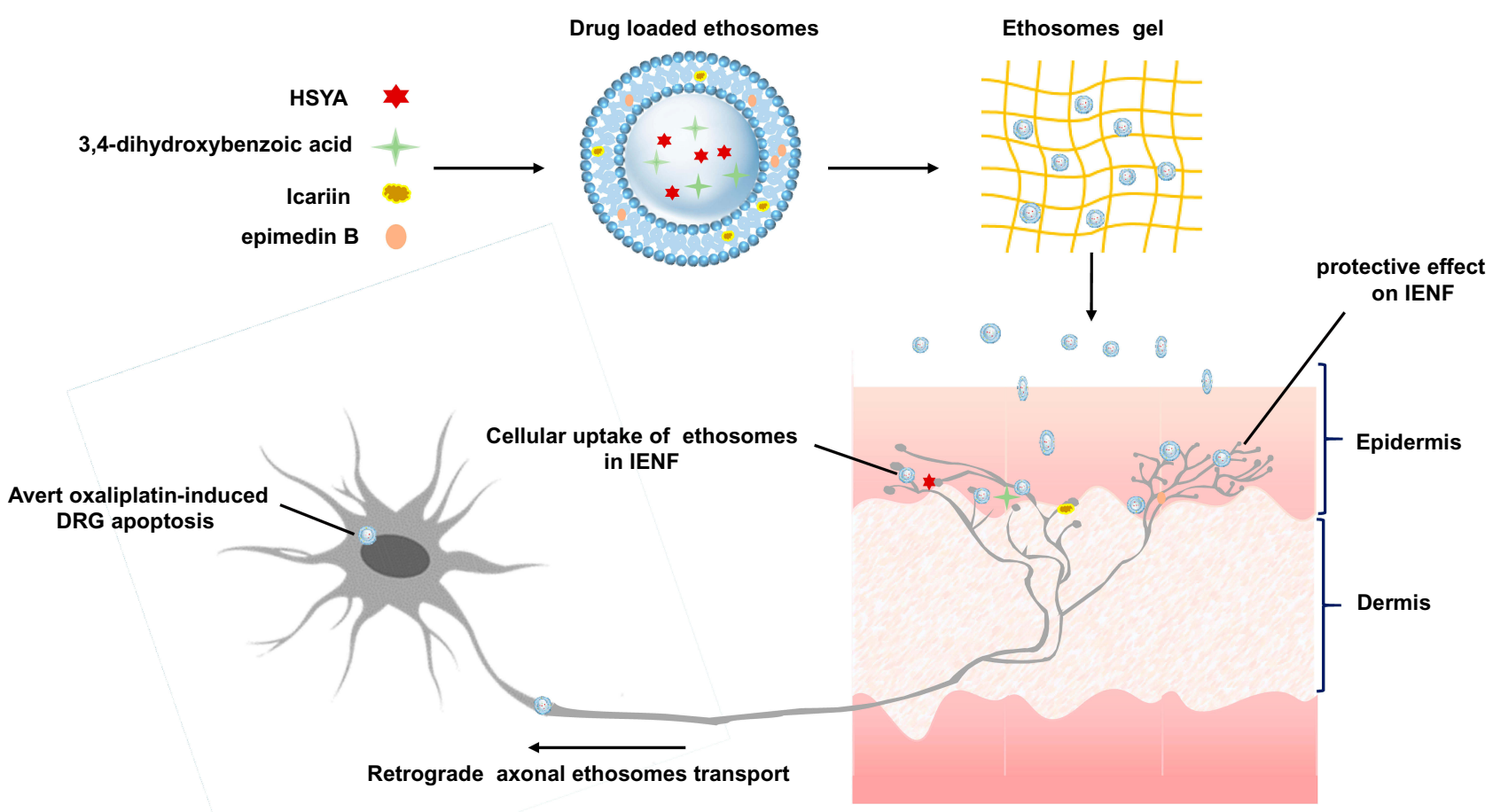

Figure I Schematic illustration of the preparation of four drug loaded ethosomes gel for the treatment of peripheral neuropathy induced by Oxaliplatin.

respectively. Two microliters of CA was selected to develop ethosomes because high concentrations of CA could induce the apoptosis of primary culture of Schwann cells in vitro.

After optimizing the amount of CA, the amounts of Phospholipon 90G, cholesterol, 70\% (v/v) ethanol and propylene glycol were optimized for prepared ethosomes (Table 2). The entrapment efficiency of all the agents in every ethosomes batch was greater than $95 \%$ (Table 4 ). The data showed that Phospholipon 90G may be an appropriate excipient for the five agents to develop ethosome

Table 3 The Cumulative Amounts of the Drugs in Receptor and Skin After the Application of the Ethosomes Formulations with Different Amount of CA $(n=3)$

\begin{tabular}{|c|c|c|c|c|c|c|}
\hline & \multirow{2}{*}{$\begin{array}{l}\text { Volume } \\
\mu \mathrm{L}\end{array}$} & \multicolumn{5}{|c|}{ The Amount of Drug $\left(\right.$ Mean $\left.\left(\mu \mathrm{g} / \mathrm{cm}^{2}\right) \pm S D\right)$} \\
\hline & & CA & DA & HSYA & EpimedinB & Icarrin \\
\hline \multirow{4}{*}{ Receptor $(\mu \mathrm{g} / \mathrm{mL})$} & 0 & - & $3.67 \pm 0.36$ & - & $0.63 \pm 0.23$ & - \\
\hline & 2 & $4.18 \pm 0.64$ & I. $32 \pm 0.42$ & - & $52.90 \pm 2.99 * *$ & - \\
\hline & 5 & $20.18 \pm 4.67$ & $2.09 \pm 0.42$ & - & $50.90 \pm 1.33^{* *}$ & - \\
\hline & 10 & $64.33 \pm 13.68$ & $3.40 \pm 0.25$ & - & $90.59 \pm 4.03 * *$ & - \\
\hline \multirow[t]{4}{*}{ Epidermis $\left(\mu \mathrm{g} / \mathrm{cm}^{2}\right)$} & 0 & & $1.19 \pm 0.29$ & $0.56 \pm 0.02$ & $1.09 \pm 0.15$ & $1.05 \pm 0.22$ \\
\hline & 2 & & $1.78 \pm 0.27 *$ & $0.72 \pm 0.03$ & $1.44 \pm 0.11 *$ & $1.01 \pm 0.10$ \\
\hline & 5 & $1.04 \pm 0.13$ & $1.26 \pm 0.21$ & $0.43 \pm 0.04$ & $2.00 \pm 0.48 * *$ & $0.83 \pm 0.20$ \\
\hline & 10 & $1.98 \pm 1.72$ & $1.12 \pm 0.08$ & $0.45 \pm 0.08$ & $1.55 \pm 0.29 *$ & $0.69 \pm 0.03$ \\
\hline \multirow[t]{4}{*}{ Dermis $\left(\mu \mathrm{g} / \mathrm{cm}^{2}\right)$} & 0 & & $0.43 \pm 0.15$ & $0.33 \pm 0.05$ & $0.85 \pm 0.22$ & $0.39 \pm 0.09$ \\
\hline & 2 & $0.44 \pm 0.14$ & $0.64 \pm 0.12^{*}$ & $0.31 \pm 0.01$ & $3.86 \pm 0.44 * *$ & $0.30 \pm 0.03$ \\
\hline & 5 & $1.46 \pm 0.83$ & $0.94 \pm 0.23^{*}$ & $0.27 \pm 0.01$ & $9.15 \pm 1.40^{* *}$ & $0.34 \pm 0.03$ \\
\hline & 10 & $5.33 \pm 0.86$ & $0.72 \pm 0.02 *$ & $0.29 \pm 0.03$ & $7.50 \pm$ I.7I** & $0.29 \pm 0.04$ \\
\hline
\end{tabular}

Notes: $* P<0.05, * * P<0.01$ vs ethosomes without cinnamaldehyde $(0 \mu \mathrm{L})$. 
Table 4 The Particle Sizes and the Entrapment Efficiencies of the Ethosomes Formulations $(n=2)$

\begin{tabular}{|c|c|c|c|c|c|c|c|}
\hline \multirow[t]{2}{*}{ Batches } & \multirow{2}{*}{$\begin{array}{l}\text { Particle Size }(\mathrm{nm}) \\
\text { Mean } \pm \text { SD }\end{array}$} & \multirow[t]{2}{*}{ PDI } & \multicolumn{4}{|l|}{ EE(\%) } & \multirow[b]{2}{*}{ CA } \\
\hline & & & DA & HSYA & EpimedinB & Icarrin & \\
\hline I & $117.5 \pm 6.4$ & $0.260 \pm 0.030$ & $96.09 \pm 0.62$ & $96.45 \pm 0.60$ & $97.89 \pm 0.34$ & $97.99 \pm 0.33$ & $97.7 I \pm 0.4 I$ \\
\hline 2 & $129.8 \pm 1.2$ & $0.237 \pm 0.000$ & $98.31 \pm 0.00$ & $98.80 \pm 0.03$ & $99.00 \pm 0.01$ & $98.92 \pm 0.01$ & $98.98 \pm 0.01$ \\
\hline 3 & $87.7 \pm 4.9$ & $0.195 \pm 0.021$ & $98.22 \pm 0.12$ & $98.72 \pm 0.09$ & $98.93 \pm 0.03$ & $98.86 \pm 0.03$ & $98.91 \pm 0.15$ \\
\hline 4 & $128.7 \pm 1.9$ & $0.229 \pm 0.022$ & $95.92 \pm 0.09$ & $96.70 \pm 0.18$ & $97.80 \pm 0.02$ & $97.82 \pm 0.04$ & $97.56 \pm 0.02$ \\
\hline 5 & $121.9 \pm 0.6$ & $0.247 \pm 0.003$ & $95.42 \pm 0.25$ & $95.49 \pm 0.56$ & $97.42 \pm 0.26$ & $97.5 I \pm 0.35$ & $97.5 I \pm 0.17$ \\
\hline 6 & $83.5 \pm 8.3$ & $0.184 \pm 0.007$ & $98.13 \pm 0.21$ & $98.66 \pm 0.21$ & $98.87 \pm 0.15$ & $98.84 \pm 0.15$ & $98.93 \pm 0.12$ \\
\hline 7 & $97.3 \pm 4.0$ & $0.140 \pm 0.008$ & $97.90 \pm 0.33$ & $98.50 \pm 0.16$ & $98.77 \pm 0.19$ & $98.65 \pm 0.23$ & $98.70 \pm 0.18$ \\
\hline 8 & $166.0 \pm 8.8$ & $0.256 \pm 0.004$ & $96.83 \pm 0.41$ & $97.35 \pm 0.28$ & $98.10 \pm 0.28$ & $98.09 \pm 0.32$ & $98.16 \pm 0.16$ \\
\hline 9 & $127.4 \pm 6.6$ & $0.285 \pm 0.011$ & $96.37 \pm 0.55$ & $96.86 \pm 0.27$ & $98.08 \pm 0.38$ & $98.07 \pm 0.10$ & $97.94 \pm 0.06$ \\
\hline 10 & $132.8 \pm 9.5$ & $0.180 \pm 0.008$ & $98.05 \pm 0.17$ & $98.54 \pm 0.12$ & $98.84 \pm 0.16$ & $98.27 \pm 0.14$ & $99.32 \pm 0.08$ \\
\hline II & $139.0 \pm 8.5$ & $0.166 \pm 0.005$ & $97.96 \pm 0.10$ & $98.57 \pm 0.09$ & $98.95 \pm 0.09$ & $98.18 \pm 0.11$ & $99.38 \pm 0.05$ \\
\hline 12 & $230.0 \pm 5.7$ & $0.236 \pm 0.025$ & $97.18 \pm 0.04$ & $97.59 \pm 0.07$ & $98.82 \pm 0.05$ & $96.77 \pm 0.09$ & $99.04 \pm 0.04$ \\
\hline
\end{tabular}

formulations by $70 \%(\mathrm{v} / \mathrm{v})$ ethanol. The particle sizes of all ethosome formulations were significantly decreased after sonication, except batch 12. Therefore, skin deposition served as the major parameter to select the optimum formulation(s).

The ethosomes formulations displaying maximum drug deposition in the epidermis were selected and prioritized due to the OXL-induced loss of nerve fibers located between the granular layer and basal layer. The amount of icarrin that accumulated in the epidermis treated with Batch 6 was significantly higher than in the samples treated with the other formulations (Table 5). The formulation of Batch 6 also displayed the maximum deposition of HSYA and epimedin B and a relatively higher amount of DA in epidermis $5.72 \pm 0.75 \mu \mathrm{g} /$ $\mathrm{cm}^{2}, 1.97 \pm 0.27 \mu \mathrm{g} / \mathrm{cm}^{2}$ and $9.25 \pm 1.21 \mu \mathrm{g} / \mathrm{cm}^{2}$, respectively. Only three batches of ethosome formulations delivered icarrin to the dermis. The maximum amount of icarrin $(0.77 \pm 0.13 \mu \mathrm{g} /$ $\mathrm{cm}^{2}$ ) was detected in dermis after applying Batch 6 for 24 h. However, HSYA and icarrin were not be detected in the receptor compartment after treatment with all batches, indicating that the intact ethosomes formulations developed in this experiment achieved drug targeting to the skin. Although the amount of DA and Epimedin B that penetrated the skin after delivery by Batch 6 were not the highest, they still protected Schwann cells and DRG neurons from OXL-induced injury in the in-vitro cell culture experiment in our previous studies. Therefore, Batch 6 was selected to evaluate the release behavior and was prepared as an ethosomes gel containing $180 \mathrm{mg}$ Phospholipon 90G, $2.4 \mathrm{~mL}$ of ethanol, $0.6 \mathrm{~mL}$ of propylene glycol and $2 \mu \mathrm{L}$ of CA. Furthermore, CA potentially mimicked cholesterol by inserting in the lipoid bilayers, increasing the deformability and integrity of ethosomes, because Batch 6 did not contain cholesterol.

\section{Preparation and Optimization of Ethosomes Gels}

The rheological behavior and spreadability of all ethosomes gel formulations were evaluated to select the suitable carbopol grade and concentration. The steady shear measurements showed a decreased viscosity, followed by an increased shear rate of all the ethosomes gel formulations, indicating that they belong displayed Non-Newtonian flow with shear thinning behavior (Figure 2A). The Ostwald model was employed to further analyze the index of flow (n), which was used to determine the extent deviated from the Newtonian flow of the gel formulations. The flow index of all the ethosomes gel formulations was less than 1, representing the pseudoplastic flow with shear-thinning behavior of all the gel formulations (Table 6).

The spreading area of all the gel formulations increased as the weight of increased (Figure 2B), indicating that all the gel formulations displayed good spreadability that would increase their ease of use. The gel formulation prepared with $0.4 \%$ Carbopol 940 had a higher $\mathrm{S} f$ value (0.37) and a lower viscosity than Carbopol 980. The topical gel formulation should exhibit a proper spreadability and a higher viscosity to allow a sufficient time for drug delivery to the skin by prolonging the skin retention time. Therefore, the gel formulations with a high viscosity were selected to evaluate the viscoelasticity and stability. 


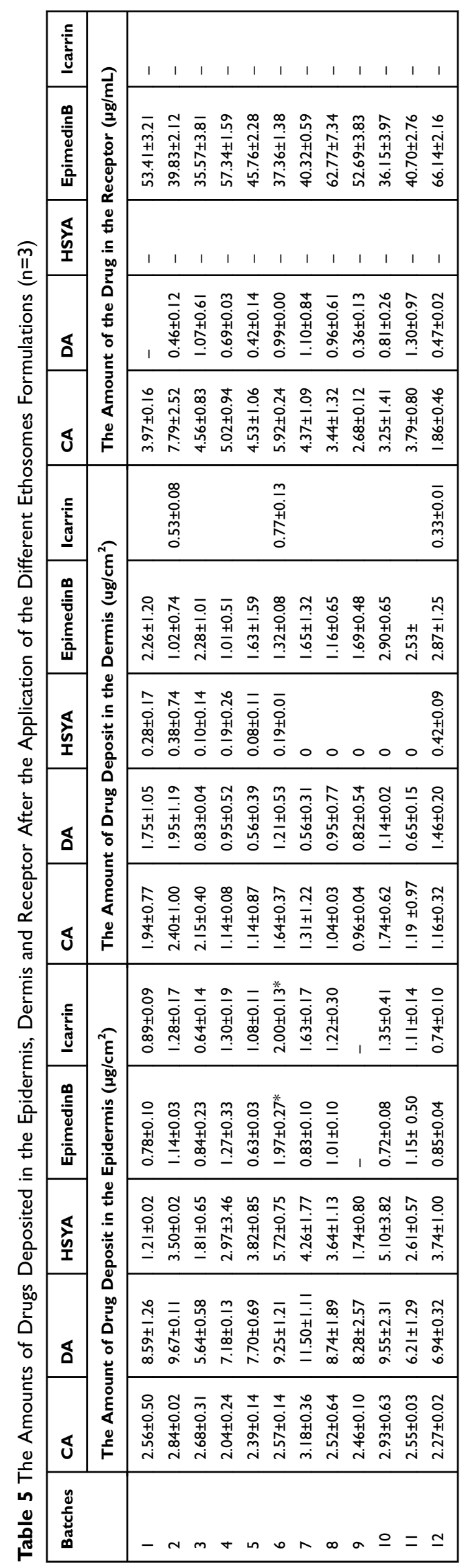

The value of G' (storage modulus) was higher than the value of G" (loss modulus) in the high-frequency region of the ethosomes gels prepared with $0.5 \%$ Carbopol 940 (Figure 2C). The complex viscosity of the loss modulus during deformation was approximately zero due to the hardening of the gel after dehydration. The ethosomes gels prepared with $0.5 \%$ carbopol hardened due to dehydration induced by high temperatures during long-term storage. We speculated that the ethosome dispersion altered the crosslinking of Carbopol 940 and resulted in a poor stability. In the low-frequency region, the magnitude of $G$ ' was greater than the magnitude of G" over a wide frequency range of the ethosomes gel prepared with $0.4 \%$ Carbopol 980, similar to the gels prepared with $0.5 \%$ Carbopol 980 (Figure 2D and E). These findings supported the observations of better viscoelastic properties and better stability of those two gels. In the high-frequency region, the magnitude of G" increased was higher than G' of the two gels, indicating that the gels displayed a better elasticity. ${ }^{35}$ Furthermore, the topical gel formulation should exhibit a proper spreadability and a higher viscosity to ensure a sufficient time for drug delivery to the skin by prolonging the skin retention time. Hence, the ethosomes gel prepared with $0.5 \%$ Carbopol 980 was the optimum formulation due to its higher value of complex viscosity 4640 (Pa.s) than the ethosomes gel prepared with 0.4\% Carbopol 980 (3480 Pa.s).

\section{Characterization of Ethosomes Gel Morphology}

TEM images showed a small and uniform vesicle size of the ethosomes was, but the shape of the ethosomes was not smooth (Figure 3A), after the ethosomes were extruded from the net structure of the carbopol gel. The images also provided the evidence of the deformability of the ethosomes.

\section{In vitro Drug Release Studies}

The release behaviors of HSYA, icariin, epimedin B, DA and $\mathrm{CA}$ from ethosomes suspensions and ethosomes gels were examined over $24 \mathrm{~h}$ (Figure 3B and C). The release profiles of the five drugs from ethosomes suspensions and ethosomes gels were similar. The presence of the carbopol gel did not delay the release rate of the five drugs loaded in the ethosomes. DA and CA exhibited relatively higher release rate than the other drugs. Approximately $86 \%$ of DA and $81 \%$ of CA were released from ethosomes suspensions after $24 \mathrm{~h}$. Both DA and $\mathrm{CA}$ are micromolecular agents, with a better solubility in $2 \%$ Brij 58 solution. This may explain the higher release rate. Although HSYA and epimedin B displayed good water 
A
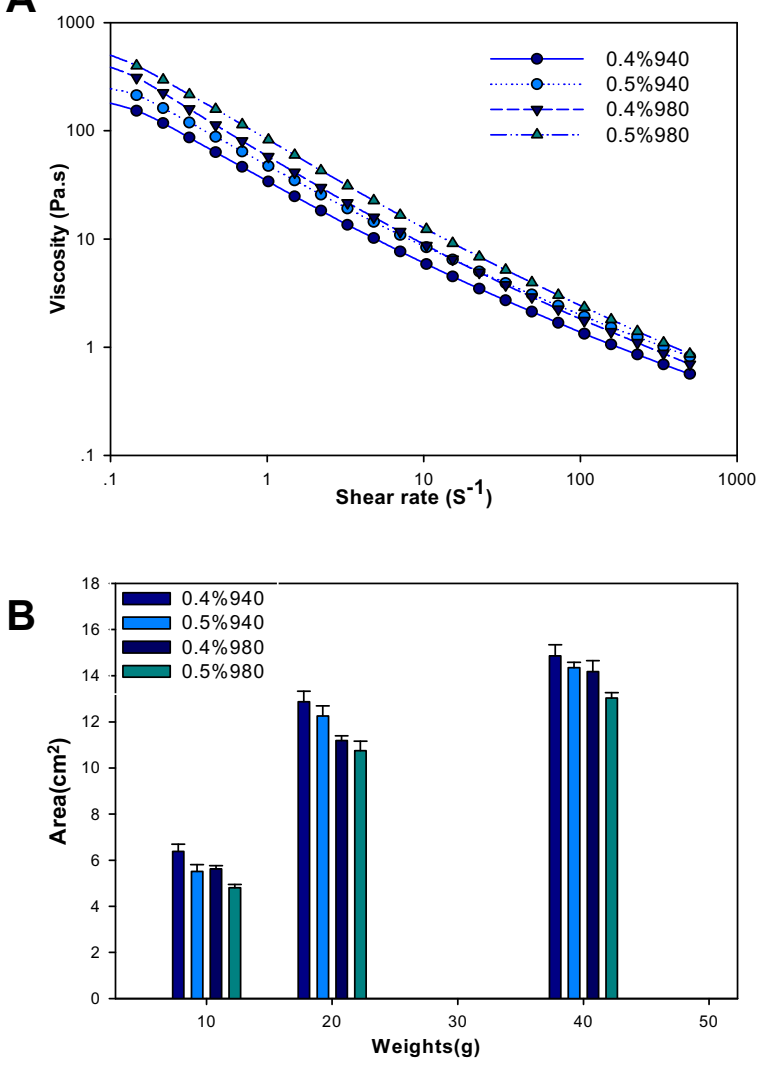

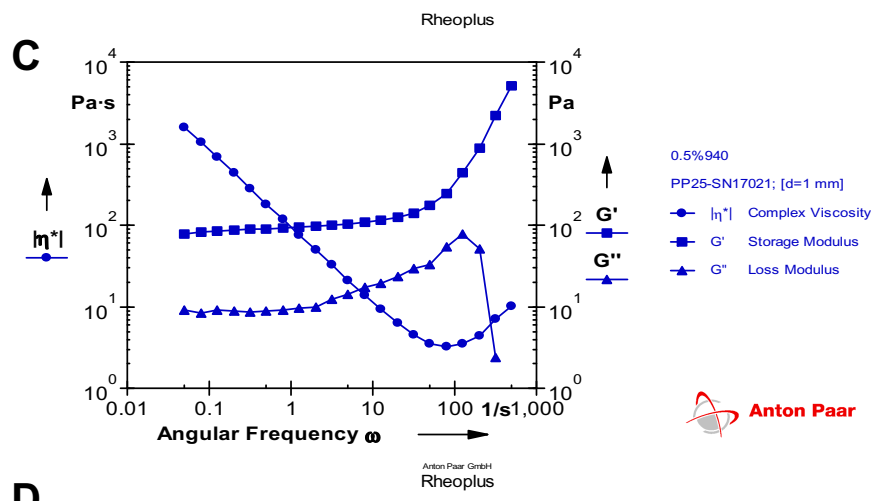

D

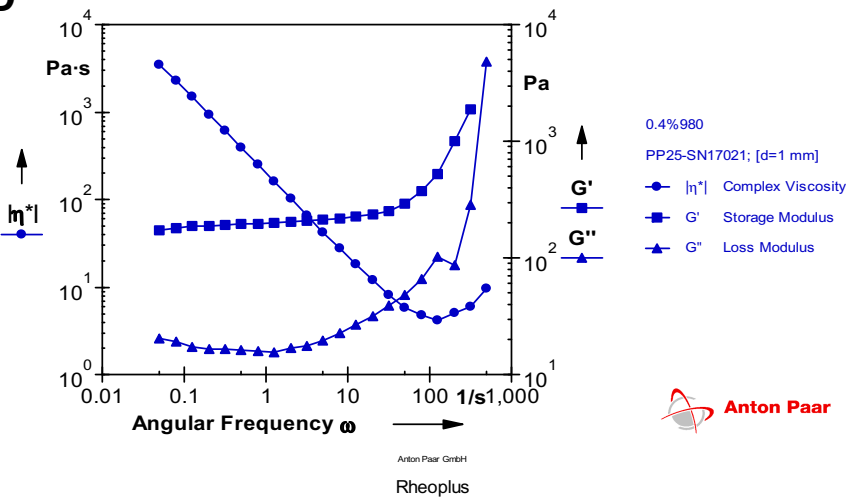

$\mathbf{E}$

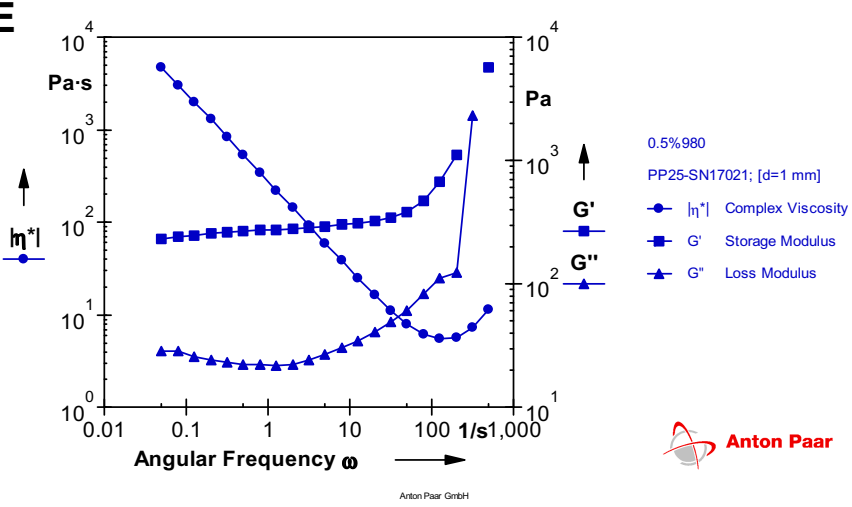

Figure 2 The spreadability and rheological property of different ethosomes gel.

Notes: (A) Viscosity-shear rate curves of different ethosomes gel. (B) The spreadabilities of different ethosomes gel. (C) The rheological property of ethosomes gel prepared with $0.5 \%$ carbopol 940 . (D) The rheological property of ethosomes gel prepared with $0.4 \%$ carbopol 980 . (E) The rheological property of ethosomes gel prepared with $0.5 \%$ carbopol 980 .

solubility, they exhibited a relatively lower amount of drug released from the ethosome suspensions drug at $24 \mathrm{~h}(59 \%$ HSYA and $57 \%$ epimedin B) due to their macromolecular

Table 6 The Parameter of the Ostwald Equation Fitted by Different Ethosomes Gel

\begin{tabular}{|l|l|l|l|}
\hline Gel & K(Pa.s $\left.{ }^{\mathbf{n}}\right)$ & Flow Index(n) & $\mathbf{R}^{\mathbf{2}}$ \\
\hline $0.4 \% 940$ & 34.675 & 0.300 & 0.975 \\
$0.5 \% 940$ & 48.477 & 0.307 & 0.977 \\
$0.4 \% 980$ & 60.144 & 0.241 & 0.963 \\
$0.5 \% 980$ & 83.051 & 0.231 & 0.977 \\
\hline
\end{tabular}

structures. After $24 \mathrm{~h}$, only 19\% icarrin was released from ethosomes suspensions, similar to the amount released from the ethosomes gel. The poor solubility and large structure of the icariin molecule resulted in a slow-release rate.

\section{In vitro Drug Deposition Studies}

Only epimedin B and CA were detected in the receptor compartment after the application of the ethosome gel, consistent with the results of the in vitro skin deposition studies using the ethosomes formulation (Figure 3D). A potential explanation for these findings is that some ethosomes were broken on the surface of the stratum corneum and released the CA, which increased the cumulative amounts of EpimedinB across that 

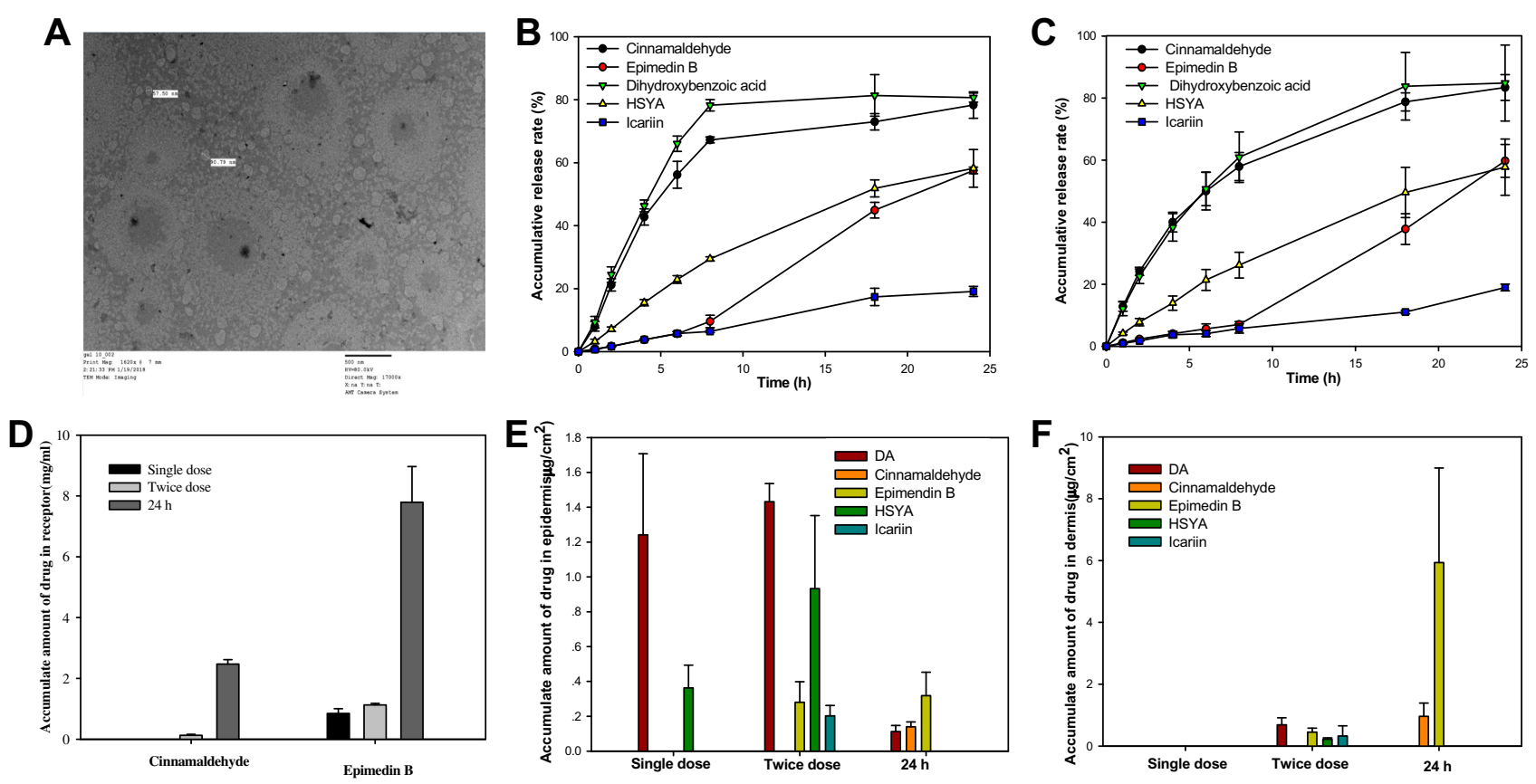

Figure 3 Characterization of the optimized ethosomes gel.

Notes: (A) The morphology of the optimized ethosomes gel by TEM. (B) The release curves of five drugs in ethosomes formulation. (C) The release curves of five drugs in ethosomes gel formulation. The cumulative amounts of drugs in receptor (D), epidermis (E) and dermis (F) after the application of ethosomes gel by different methods.

penetrated the skin and ensured the protection of DRG neurons after transport through the circulation.

The cumulative amounts of drugs that were deposited in the epidermis after the administration of the ethosomes gel were shown in (Figure 3E). All drugs loaded in the ethosomes, except CA, were detected in the epidermis after applying a single dose of the ethosomes gel although the concentrations of epimedin B and icariin were below the limit of quantitation. When the skin samples were rubbed with the ethosome gel twice, the amounts of the four drugs that accumulated in the epidermis increased. Thus, some intact ethosomes vesicle were delivered into the deep layers of skin, and less CA was evaporated during the sample analysis procedure. Furthermore, an increase in the application frequency might increase the cumulative amounts of the drugs that were deposited in the skin. However, HSYA and icariin were not detected in the epidermis after the continuous application of excess ethosomes gel for $24 \mathrm{~h}$.

The intact ethosomes vesicles tended to be transported to the deep layers of skin as water evaporated from the ethosomes gel when a small amount of gel was applied on the skin. ${ }^{36,37}$ However, a sufficient amount of water remained in the ethosomes gel when an excess amount was applied on the surface of the stratum corneum. We speculated that ethosomes did not penetrate the stratum corneum as intact vesicle when an excess amount of the ethosomes gel formulation was applied. The cumulative amounts of the drugs that deposited in the dermis are shown in Figure $3 \mathrm{~F}$ and further confirm this hypothesis. An insufficient drugs on the skin following the application of a single dose of the ethosomes gel resulted in a lack of detection of drugs in the dermis after $24 \mathrm{~h}$. All the drugs, except CA, were detected in the dermis when the ethosome gel was applied twice.

Based on these results, we concluded that an increase in the retention time of drugs on skin would delay the evaporation of water and provide sufficient time for ethosomes to be delivered into the deep layers of skin. Meanwhile, the amounts of drugs retained in skin increased with the increased frequency of administration. Hence, the gel was applied two times a day to evaluate the efficacy of the gel.

\section{Skin Permeation Studies Using FITC and Rhodamine B}

The hydrophobic nature, solubility and $\log \mathrm{P}$ values were all considered in the selection of the dye used for skin distribution studies. The $\log \mathrm{P}$ value of FITC was 4.8 and used to study the behaviors of icarrin, CA and epimedin B. Rhodamine B is a hydrophilic dye, and its molecular weight (479.0) is between the molecular weights of DA (154.1) and HSYA (612.5). Hence, rhodamine B was selected to study the distribution of DA and HSYA in skin. 
Confocal images of vertical skin sections treated with the control and dye-loaded ethosomes gels are presented in Figure 4. The florescence of FITC and rhodamine B was observed in the stratum corneum, viable epidermis and dermis of the skin after the dye-loaded ethosomes gel was applied twice. After the application of a single dose, the florescence of FITC and rhodamine B was only observed in the stratum corneum. However, rhodamine B only penetrated to the granular layer and FITC was weakly detected in the dermis of the control group. These results may be attributed to the high $\log \mathrm{P}$ value of FITC, which was more suitable for transdermal penetration.

High florescence intensity of FITC was observed in the dermis when the skin was treated with an excess amount of the dye-loaded ethosomes gel for $24 \mathrm{~h}$. This finding further confirmed the hypothesis proposed based on the results of the in vitro drug deposition studies. Some of the ethosomes were broken as they penetrated through the stratum corneum, while rhodamine B and FITC were released. FITC penetrated

into the deep layer of skin and displayed a higher florescence intensity than rhodamine $\mathrm{B}$ in the dermis due to its high $\log \mathrm{P}$ value. FITC and rhodamine B did not completely reflect the skin distribution of the drugs loaded in the ethosomes gel because the molecular weights of FITC and rhodamine B were lower than icarrin, epimedin B and HSYA. FITC presented a higher $\log \mathrm{P}$ value than icarrin and epimedin B. However, the results of the skin permeation studies using FITC and rhodamine B indicated that an increased frequency of administration might increase drug deposition in the skin.

\section{Neuroprotective Efficacy Behavior Test}

The sensitivity of rats to a mechanical stimulus that normally does not provoke pain was evaluated using von Frey filaments. The response to mechanical stimulation was evaluated by assessing paw withdrawals (Figure 5A). Compared to the control group, rats in the model group

Continuous 24h
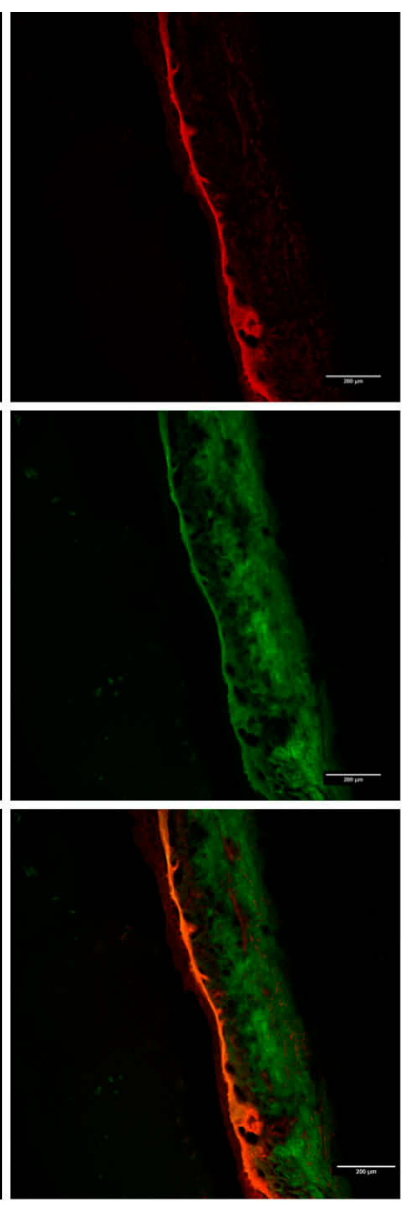

Control

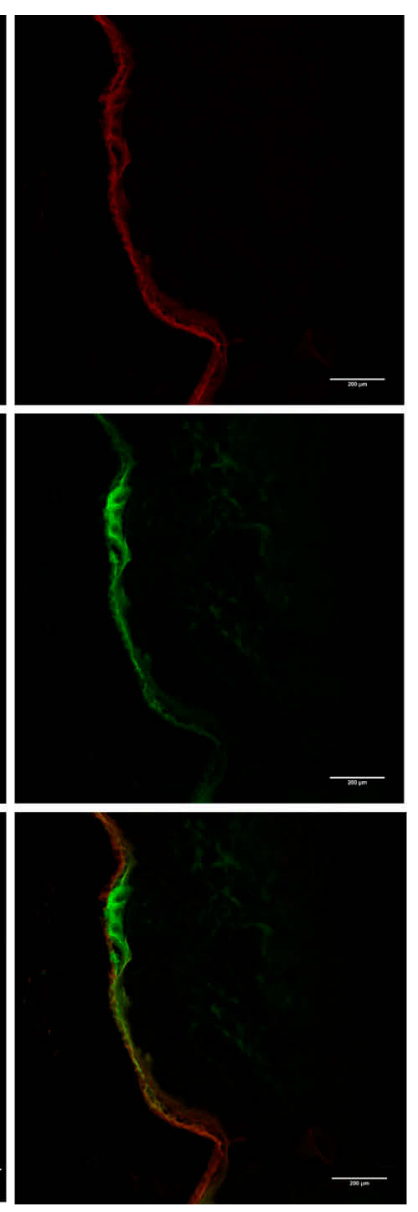

Figure 4 The fluorescent expression of the skin after the application of the ethosomes gel by a different method. 

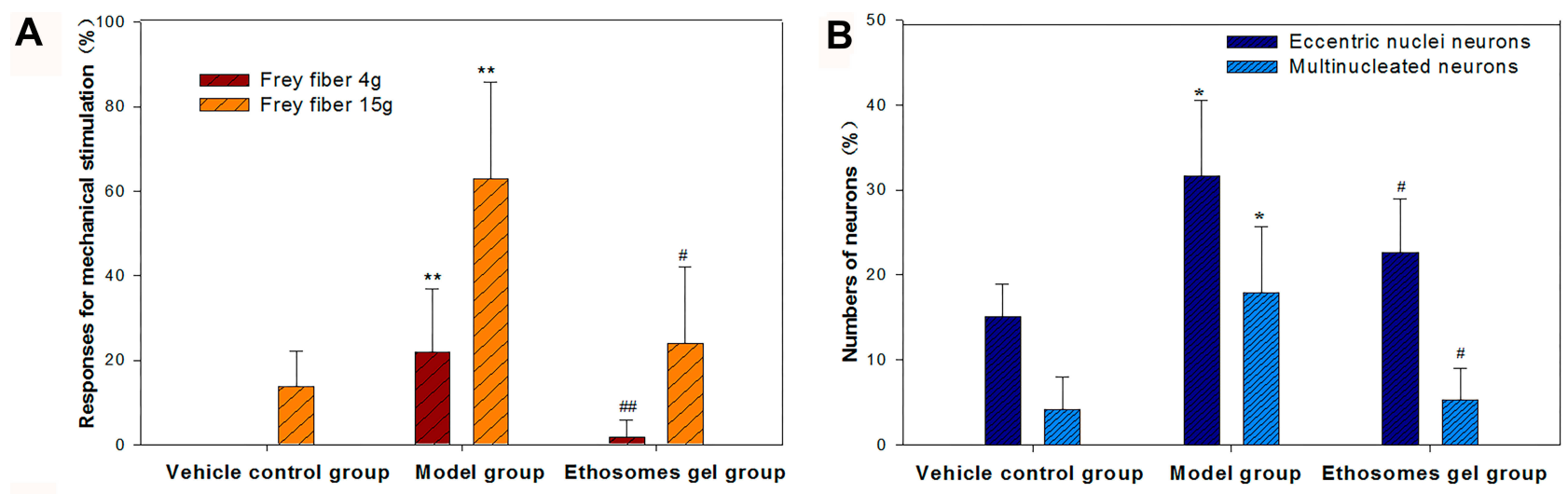

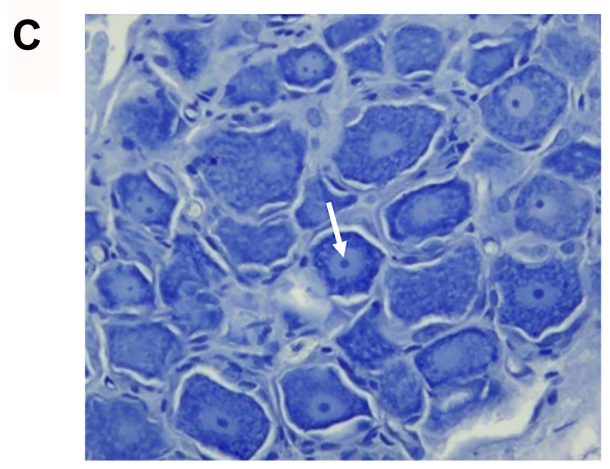

Vehicle control group

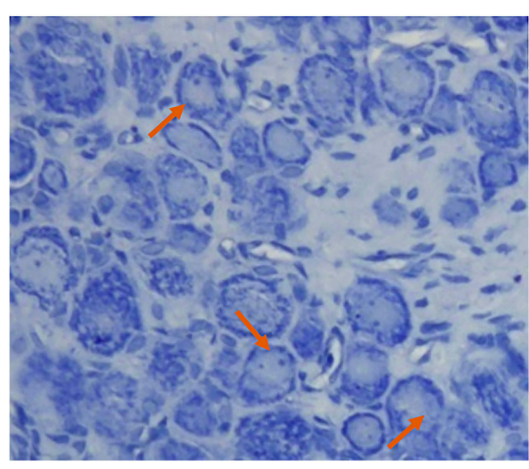

Model group

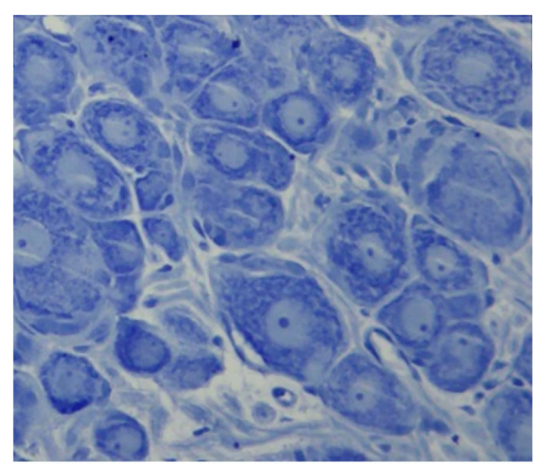

Ethosomes gel group

Figure 5 The neuroprotective effect on rat in vivo.

Notes: (A) Behavior test, the response to mechanical stimulation in different groups $\left({ }^{* * P}<0.01\right.$ vs vehicle control group; ${ }^{\#} P<0.05$, ${ }^{\# \#} P<0.0$ I vs model group). (B) The numbers of eccentric nuclei and multinucleated neurons in different groups ( ${ }^{*} P<0.05$ vs vehicle control group; ${ }^{\#} P<0.05$ vs model group). (C) The morphology of normal nucleoli (white arrow), eccentric nuclei and multinucleated neurons (yellow arrows) of DRG neurons.

showed an obvious increase in the number of paw withdrawals after treatment with both $4 \mathrm{~g}$ and $15 \mathrm{~g}$ von-Frey fibers. Thus, repeated treatment with OXL induced mechanical allodynia and hyperalgesia in rats. The percentage of paw withdrawals of rats in the ethosomes gel group was only $1.7 \pm 4.1 \%$ after the rats were stimulated with $4 \mathrm{~g}$ von-Frey fibers, a value that was significantly different from the model group. It indicated that the drugs in the ethosomes gel protected the DRG neurons and peripheral nerves from injury by OXL. To the morphology of DRG neurons was examined to further confirm this hypothesis.

\section{Morphology of DRG Neurons}

The accumulation of OXL in DRG neurons was the main cause of the OXL-induced mechanical allodynia, resulting in increased numbers of eccentric nuclei and multinucleated neurons. As shown in Figure 5C, the nuclei and nucleoli of normal neurons, which were relatively large, were located in the center of cells and are indicated by the white arrow. The DRG neurons from the rats treated with OXL showed significantly increased numbers of eccentric nuclei and multinucleated neurons compared to the control group (Figure 5B). In ethosomes gel group, the numbers of eccentric nuclei were significantly decreased, suggesting that the drugs loaded in the ethosomes gel exerted a protective effect on the DRG neurons.

\section{Conclusion}

In the present study, a novel topical delivery system containing four active drugs with different lipophilicity values and molecular weights were established by preparing an ethosomes gel. Epimedin B and icarrin were loaded in the lipid bilayers of ethosomes, and HSYA and DA were hydrophilic drugs, that were entrapped inside water core of ethosomes. CA functioned as a cholesterol mimic that was inserted into the lipid bilayers and increased penetration of the ethosomes. The ethosomes gel with desirable spreadability, viscoelasticity and fast release of drugs delivered the four active drugs to the deep layers of skin where the IENF was located following a topical application twice a day. The ethosomes gel co-loaded with the four drugs counteracted the 
mechanical allodynia and hyperalgesia induced by OXL and protected against OXL-induced toxicity in DRG neurons in vivo, displaying neuroprotective efficacy. Our study supplied a novel therapeutic method to treat OIPN. Therefore, clinical studies aiming to further investigate the drug coloaded ethosomes gel as a potential neuroprotective agent to prevent OIPN are necessary.

\section{Abbreviations}

OXL, oxaliplatin; HSYA, hydroxysafflor yellow A; DA, 3, 4-dihydroxybenzoic acid; CA, cinnamaldehyde; DRG, dorsal root ganglion; SCs, Schwann cells; WLT, WenLuo-Tong; OIPN, oxaliplatin-induced peripheral neuropathy; IENF, intra epidermal nerve fibers.

\section{Acknowledgment}

The authors are grateful to Professor Bozena MichniakKohn (Center for Dermal Research (CDR) \& Laboratory for Drug Delivery (LDD), Rutgers-The State University of New Jersey) for providing technical assistance.

\section{Disclosure}

The authors report no conflicts of interest in this work.

\section{References}

1. Areti A, Komirishetty P, Kumar A. Carvedilol prevents functional deficits in peripheral nerve mitochondria of rats with oxaliplatin-evoked painful peripheral neuropathy. Toxicol Appl Pharmacol. 2017;322:97-103. doi:10.1016/j.taap.2017.03.009

2. Zedan AH, Hansen TF, Svenningsen ÅF, Vilholm OJ. OxaliplatinInduced neuropathy in colorectal cancer: many questions with few answers. Clin Colorectal Cancer. 2014;13(2):73-80. doi:10.1016/j. clcc.2013.11.004

3. Sereno M, Gutiérrez-gutiérrez G, Gómez-raposo C, et al. Oxaliplatin induced-neuropathy in digestive tumors. Crit Rev Oncol Hematol. 2014;89(1):166-178. doi:10.1016/j.critrevonc.2013.08.009

4. Kawashiri T, Egashira N, Watanabe $\mathrm{H}$, et al. Prevention of oxaliplatin-induced mechanical allodynia and neurodegeneration by neurotropin in the rat model. Eur J Pain. 2011;15(4):344-350. doi:10.1016/j.ejpain.2010.08.006

5. Persson A-K, Hoeijmakers JGJ, Estacion M, Black JA, Waxman SG. Sodium channels, mitochondria, and axonal degeneration in peripheral neuropathy. Trends Mol Med. 2016;22(5):377-390. doi:10.1016/j. molmed.2016.03.008

6. Cerqueira SR, Lee Y-S, Cornelison RC, et al. Decellularized peripheral nerve supports Schwann cell transplants and axon growth following spinal cord injury. Biomaterials. 2018;177:176-185. doi:10.1016/j. biomaterials.2018.05.049

7. Rosenberg LH, Cattin A-L, Fontana X, et al. HDAC3 regulates the transition to the homeostatic myelinating schwann cell state. Cell Rep. 2018;25(10):2755-2765.e2755. doi:10.1016/j.celrep.2018.11.045

8. Fondello C, Agnetti L, Villaverde MS, Simian M, Glikin GC, Finocchiaro LME. The combination of bleomycin with suicide or interferon- $\beta$ gene transfer is able to efficiently eliminate human melanoma tumor initiating cells. Biomed Pharmacother. 2016;83:290-301. doi:10.1016/j.biopha.2016.06.038
9. Liu Y, Feng N. Nanocarriers for the delivery of active ingredients and fractions extracted from natural products used in traditional Chinese medicine (TCM). Adv Colloid Interface Sci. 2015;221:60-76. doi:10.1016/j.cis.2015.04.006

10. Lin HM, Lin LF, Xia ZZ, et al. Neuroprotective effects and UPLC-Q-TOF/MS-based active components identification of external applied a novel Wen-Luo-Tong microemulsion. Artif Cell Nanomed Biotechnol. 2018;46(8):1981-1991.

11. Deng B, Jia LQ, Pan L, et al. Wen-Luo-Tong prevents glial activation and nociceptive sensitization in a rat model of oxaliplatin-induced neuropathic pain. Evidence Based Complementary Altern Med. 2016;2016:1-7. doi:10.1155/2016/3629489

12. Zhang Y, Yin L, Zheng N, et al. Icariin enhances remyelination process after acute demyelination induced by cuprizone exposure. Brain Res Bull. 2017;130:180-187. doi:10.1016/j.brainresbull.2017.01.025

13. Jin J, Wang H, Hua X, Chen D, Huang C, Chen Z. An outline for the pharmacological effect of icariin in the nervous system. Eur J Pharmacol. 2019;842:20-32. doi:10.1016/j.ejphar.2018.10.006

14. Liu B, Zhang H, Xu C, et al. Neuroprotective effects of icariin on corticosterone-induced apoptosis in primary cultured rat hippocampal neurons. Brain Res. 2011;1375:59-67. doi:10.1016/j.brainres.2010. 12.053

15. Wu L, Du Z-R, Xu A-L, et al. Neuroprotective effects of total flavonoid fraction of the Epimedium koreanum Nakai extract on dopaminergic neurons: in vivo and in vitro. Biomed Pharmacother. 2017;91:656-663. doi:10.1016/j.biopha.2017.04.083

16. Lv Y, Qian Y, Fu L, Chen X, Zhong H, Wei X. Hydroxysafflor yellow A exerts neuroprotective effects in cerebral ischemia reperfusion-injured mice by suppressing the innate immune TLR4-inducing pathway. Eur J Pharmacol. 2015;769:324-332. doi:10.1016/j.ejphar.2015.11.036

17. Xing M, Sun Q, Wang Y, Cheng Y, Zhang N. Hydroxysafflor yellow A increases BDNF and NMDARs in the hippocampus in a vascular dementia rat model. Brain Res. 2016;1642:419-425. doi:10.1016/j. brainres.2016.04.030

18. Ban JY, Cho SO, Jeon S-Y, Bae K, Song K-S, Seong YH. 3,4-dihydroxybenzoic acid from Smilacis chinae rhizome protects amyloid $\beta$ protein (25-35)-induced neurotoxicity in cultured rat cortical neurons. Neurosci Lett. 2007;420(2):184-188. doi:10.1016/j.neulet. 2007.05.009

19. Ko M-H, Hu M-E, Hsieh Y-L, Lan C-T, Tseng T-J. Peptidergic intraepidermal nerve fibers in the skin contribute to the neuropathic pain in paclitaxel-induced peripheral neuropathy. Neuropeptides. 2014;48(3):109-117. doi:10.1016/j.npep.2014.02.001

20. Chowdary PD, Che DL, Kaplan L, et al. Nanoparticle-assisted optical tethering of endosomes reveals the cooperative function of dyneins in retrograde axonal transport. Sci Rep. 2015;5:431-439.

21. Poon WW, Blurton-jones $\mathrm{M}, \mathrm{Tu} \mathrm{CH}$, et al. $\beta$-amyloid impairs axonal BDNF retrograde trafficking. Neurobiol Aging. 2011;32(5):821-833. doi:10.1016/j.neurobiolaging.2009.05.012

22. Nabhan JF, Wood KM, Rao VP, et al. Intrathecal delivery of frataxin mRNA encapsulated in lipid nanoparticles to dorsal root ganglia as a potential therapeutic for Friedreich's ataxia. Sci Rep. 2016;6:10. doi:10.1038/srep20019

23. Shao M, Hussain Z, Thu HE, et al. Drug nanocarrier, the future of atopic diseases: advanced drug delivery systems and smart management of disease. Colloids Surf B. 2016;147:475-491. doi:10.1016/j. colsurfb.2016.08.027

24. Lin HM, Michniak-kohn B, Xia ZZ, et al. Transdermal delivery of compounds with different lipophilicity and molecular weight from W/O microemulsions analyzed by UPLC-Q-TOF/MS and LC-MS/ MS. Curr Drug Deliv. 2018;15(7):1009-1019. doi:10.2174/1567201 815666171221125411

25. Wang N, Fu Q, Yang G. Determination of the solubility, dissolution enthalpy and entropy of icariin in water, ethanol, and methanol. Fluid Phase Equilib. 2012;324:41-43. doi:10.1016/j.fluid.2012. 03.022 
26. Marto J, Vitor C, Guerreiro A, et al. Ethosomes for enhanced skin delivery of griseofulvin. Colloids Surf B. 2016;146:616-623. doi:10.1016/j.colsurfb.2016.07.021

27. Ashtikar M, Nagarsekar K, Fahr A. Transdermal delivery from liposomal formulations - evolution of the technology over the last three decades. J Controlled Release. 2016;242:126-140. doi:10.1016/j. jconrel.2016.09.008

28. Zhang Y-T, Shen L-N, Wu Z-H, Zhao J-H, Feng N-P. Comparison of ethosomes and liposomes for skin delivery of psoralen for psoriasis therapy. Int J Pharm. 2014;471(1):449-452. doi:10.1016/j.ijpharm. 2014.06.001

29. Kausar H, Mujeeb M, Ahad A, et al. Optimization of ethosomes for topical thymoquinone delivery for the treatment of skin acne. J Drug Deliv Sci Technol. 2019;49:177-187. doi:10.1016/j.jddst. 2018.11.016

30. Garg V, Singh H, Bhatia A, et al. Systematic development of transethosomal gel system of piroxicam: formulation optimization, in vitro evaluation, and ex vivo assessment. AAPS PharmSciTech. 2017;18 (1):58-71. doi:10.1208/s12249-016-0489-z

31. Iizhar SA, Syed IA, Satar R, Ansari SA. In vitro assessment of pharmaceutical potential of ethosomes entrapped with terbinafine hydrochloride. J Adv Res. 2016;7(3):453-461. doi:10.1016/j.jare. 2016.03.003
32. Jain A, Doppalapudi S, Domb AJ, Khan W. Tacrolimus and curcumin co-loaded liposphere gel: synergistic combination towards management of psoriasis. $J$ Controlled Release. 2016;243:132-145. doi:10.1016/j.jconrel.2016.10.004

33. Kaur A, Katiyar SS, Kushwah V, Jain S. Nanoemulsion loaded gel for topical co-delivery of clobitasol propionate and calcipotriol in psoriasis. Nanomed. 2017;13(4):1473-1482. doi:10.1016/j.nano.2017.02.009

34. Yu L, Jun-hong Y, Ye-ting S, et al. Comparative study of penetration-enhancing effect in vitro of cinnamon oil and cinnamaldehyde on ibuprofen. China J Chin Mater Med. 2018;43 (17):3493-3497. doi:10.19540/j.cnki.cjcmm.20180702.004

35. Jain S, Patel N, Madan P, Lin SS. Formulation and rheological evaluation of ethosome-loaded carbopol hydrogel for transdermal application. Drug Dev Ind Pharm. 2016;42(8):1315-1324. doi:10.3109/03639045.2015.1132227

36. Jain S, Patel N, Shah MK, Khatri P, Vora N. Recent advances in lipid-based vesicles and particulate carriers for topical and transdermal application. J Pharm Sci. 2017;106(2):423-445. doi:10.1016/j. xphs.2016.10.001

37. Elnaggar YSR, El-refaie WM, El-massik MA, Abdallah OY. Lecithin-based nanostructured gels for skin delivery: an update on state of art and recent applications. $J$ Controlled Release. 2014;180:10-24. doi:10.1016/j.jconrel.2014.02.004
International Journal of Nanomedicine

\section{Publish your work in this journal}

The International Journal of Nanomedicine is an international, peerreviewed journal focusing on the application of nanotechnology in diagnostics, therapeutics, and drug delivery systems throughout the biomedical field. This journal is indexed on PubMed Central, MedLine, CAS, SciSearch ${ }^{\mathbb{B}}$, Current Contents ${ }^{\mathbb{R}} /$ Clinical Medicine,

\section{Dovepress}

Journal Citation Reports/Science Edition, EMBase, Scopus and the Elsevier Bibliographic databases. The manuscript management system is completely online and includes a very quick and fair peer-review system, which is all easy to use. Visit http://www.dovepress.com/ testimonials.php to read real quotes from published authors. 